\title{
Myocardial viability assessment in 18FDG PET/CT' study (18FDG PET myocardial viability assessment)
}

Małgorzata Kobylecka', Joanna Mączewska',

Katarzyna Fronczewska-Wieniawska1, Tomasz Mazurek², Maria Teresa Płazińska ${ }^{1}$, Leszek Królicki ${ }^{1}$

${ }^{1}$ Nuclear Medicine Department Warsaw Medical University, Warsaw, Poland

${ }^{2}$ Chair and Department of Internal Medicine and Cardiology

Warsaw Medical University, Warsaw, Poland

\section{[Received 20 | 2012; Accepted 3 || 2012]}

\section{Abstract}

Accurate identification of viable myocardium is crucial in patient qualification for medical or surgical treatment. Only persons with confirmed cardiac viability will benefit from revascularization procedures. It is also well known, that the amount of viable myocardium assessed preoperatively is the best indicator of long term cardiac event free survival after cardiac intervention. There are several diagnostic approaches used in current clinical practice for assessment of myocardial viability. Analysis of wall thickness or myocardial contraction, evaluation of cardiac perfusion or metabolism can be assessed using following modalities: Echocardiography, Cardiac Molecular Imaging techniques (PET, SPECT), Cardiovascular MR or Cardiovascular CT. The article describes the methods and problems of viability assessment in 18FDG PET study. PET imaging has proved its accuracy and reproducibility for myocardial ischemia and viability assessment. However this unique in its ability for showing the particular substrate metabolism technique has

Correspondence to: dr n. med. Małgorzata Kobylecka (MD, PhD)

Nuclear Medicine Department Warsaw Medical University,

Warsaw, Poland

Tel.: (+48 22) 59922 70, fax: (+48 22) 5991170

e-mail:makob@amwaw.edu.pl unfortunately some disadvantages: currently achieved PET resolution is $0.4 \mathrm{~cm}$. However the combined devices multislice computed tomography scanners with PET (PET/CT) are now widely used in clinical practice. This combination allows for wider morphologic assessments: coronary calcium scoring and non-invasive coronary angiography may be added to myocardial perfusion/metabolic imaging if necessary.

Key words: cardiac viability, hibernation, perfusion, cardiac imaging, viability imaging, SPECT, MIBI-SPECT, PET, 18FDG-PET, PET/CT, perfusion/metabolic mismatch

Nuclear Med Rev 2012; 15, 1: 52-60

\section{Background}

Accurate identification of viable myocardium is crucial in patient qualification for medical or surgical treatment. Only persons with confirmed cardiac viability will benefit from revascularization procedures. On the other hand, in patients with preserved myocardial viability treated medically the annual cardiac death rate increases significantly-up to $16 \%$ versus $3.2 \%$ in the group after revascularization. A meta-analysis [1] showed $80 \%$ reduction in mortality if such patients had an interventional, revascularization treatment. It is also well known, that the amount of viable myocardium assessed preoperatively is the best indicator of long term cardiac event free survival after cardiac intervention [2]. Patients with no signs of viable myocardium should not be subjected to the risks of invasive surgery, because they don't have areas of myocardium that will show improved function after revascularization. According to the results of Allman's meta-analysis there is no significant difference noted between medical therapy and revascularization if patients had primarily nonviable myocardium (7.7\% vs $6.2 \%$ ) [1]. There are also data demonstrating that after revascularization, even in presence of myocardial viability, the clinically significant improvement in global left ventricular function is noted only in patients with relatively large areas of hibernated tissue $->17 \%$ of the left ventricular mass [3]. 


\section{Diagnostic imaging techniques for viability assessment}

There are several diagnostic approaches used in current clinical practice for assessment of myocardial viability. Analysis of wall thickness or myocardial contraction, evaluation of cardiac perfusion or metabolism can be assessed using following modalities: Echocardiography, Cardiac Molecular Imaging techniques (PET, SPECT), Cardiovascular MR or Cardiovascular CT.

\section{Stress echocardiography}

Among all cardiac visualization methods, stress echocardiography is probably still most commonly used in clinical practice for myocardial viability assessment. A rest cardiac wall thickness and contractile function can easily be assessed by 2D echocardiography. Infusion of either low dose-dobutamine or dipirydamole - a pharmacological "stress" agent - allows for the differentiation between irreversibly damaged scar tissue and viable, severely hypoperfused, dysfunctional myocardium. Furthermore, the myocardial contrast echocardiography with venous injection of contrast can also define the presence of coronary artery disease and enhance the determination of myocardial viability. The global assessment of the heart using the new $\mathrm{ECHO}$ technologies such as instantaneous full volume echo imaging will further improve diagnostic accuracy of echocardiography. Advantages of echocardiography include its relatively low cost and short time of the examination. The study is also easy to perform with widely available equipment. The disadventages include high interobserver variability and subjectivity of the interpretation. Poor visualization is the most common limitation of this technique. Diagnostic accuracy of the test appears unfortunately especially decreased in patients with severely impaired cardiac and lung function- the group who might mostly benefit from myocardial viability assessment. A meta-analysis referred to $\mathrm{ECHO}$ pharmacological stress test has shown that the sensitivity (84\%) and specificity $(81 \%)$ is decreasing while the cardiac left ventricular dysfunction increases [2].

\section{Cardiac magnetic resonance}

Cardiac magnetic resonance is a well validated method for assessment of myocardial masses and size, for quantification of ventricular function. New, fast scanning MR methods allow for the analysis of myocardial perfusion and cardiac dysfunction (at rest and during the pharmacological stress study), for detection of myocardial viability and visualization of irreversibly changed myocardium-scar tissue. Direct visualization of the scar is possible by a technique called "delayed enhancement "(DE CMR). The injected contrast medium shows the prolonged accumulation in cardiac scar tissue. The DE CMR images are obtained approximately 10 min after intravenous contrast administration. Cardiac MRI offers highest spatial resolution and has recently become the new gold standard for quantification of myocardial scar and for evaluation of myocardial viability. Its superior spatial resolution allows for visualization of small, subendocardial lesions. Because 18FDG-PET was until recently the gold standard for myocardial viability assessment, in many papers MRI techniques are judged comparatively. In most papers approximately 95\% concordance between techniques is noted [4]. MR technique appeared to be more accurate than molecular imaging especially for non-transmural scar recognition. However, despite the numerous adventages, there are also some disadventyages: the study is contraindicated in patients with metallic implants and in persons with claustrophoby. The study is relatively long, the good patient's cooperation is necessary (for example the breath holding).

\section{Cardiac CT}

Cardiac CT is currently used in clinical practice for evaluation of cardiac anatomy - mainly coronary arteries and coronary lumen atherosclerotic narrowing, for quantitative assessment of artery calcifications (Calcium Score) - which are pivotal to the prognosis of patients with coronary artery disease. The whole potential of cardiac CT has not been fully realized. Current available dual source CT scanners allow for the morphological assessment of coronary arteries and myocardial perfusion during one scan. Multidetector ECG-gated CT (MDCT) has recently become more available for routine use. MDCT allows not only for assessment of coronary artery morphology, but also ventricular morphology and function. Cardiac cells viability can be evaluated on the basis of CT residual myocardial blood flow assessment. Some studies have shown similar to DE CMR late enhancement phenomenon present in CT studies. The delayed images allow for differentiating acutely infarcted from viable myocardium. After the intravenous iodine contrast medium injection, the early MDCT images show the "cold spot" in the area supplied by the occluded coronary artery. On the second scan acquired later (about $10 \mathrm{~min}$ ) without another contrast medium injection, the relatively high contrast accumulation in comparison to surrounding healthy muscle remains in the area of hypoperfused myocardium, due to contrast washout impairment. The comparison studies demonstrated similar accuracy of CT and CMR [5]. The limitation of cardiac CT include low temporary resolution - which is improving but yet still limiting functional studies.

\section{Cardiac molecular imaging techniques}

Both SPECT and PET techniques allow for the identification of ischemic but viable myocardium, analyzing myocardial perfusion and function (in gated exams). PET may also be used to evaluate myocardial metabolism. The thallium redistribution SPECT studies present 201TI uptake depending on cell membrane integrity and thus myocyte viability. Additionally, the initial uptake of radiopharmaceutical shows the regional perfusion, allowing for thallium stress perfusion study, and assessment of coronary perfusion reserve. The sensitivity of the test is high (88\%), although the meta-analysis has shown that the test may overestimate the viability (specificity of $49 \%$ ) [2].

Over the last years FDG PET was the gold standard for myocardial metabolism assessment. This technique requires comparison of myocardial perfusion (using a perfusion PET or SPECT) with functional metabolic images obtained with FDG. The myocardial perfusion and metabolic uptake pattern is analyzed and compared. The mismatch between perfusion and function — lack of perfu- 


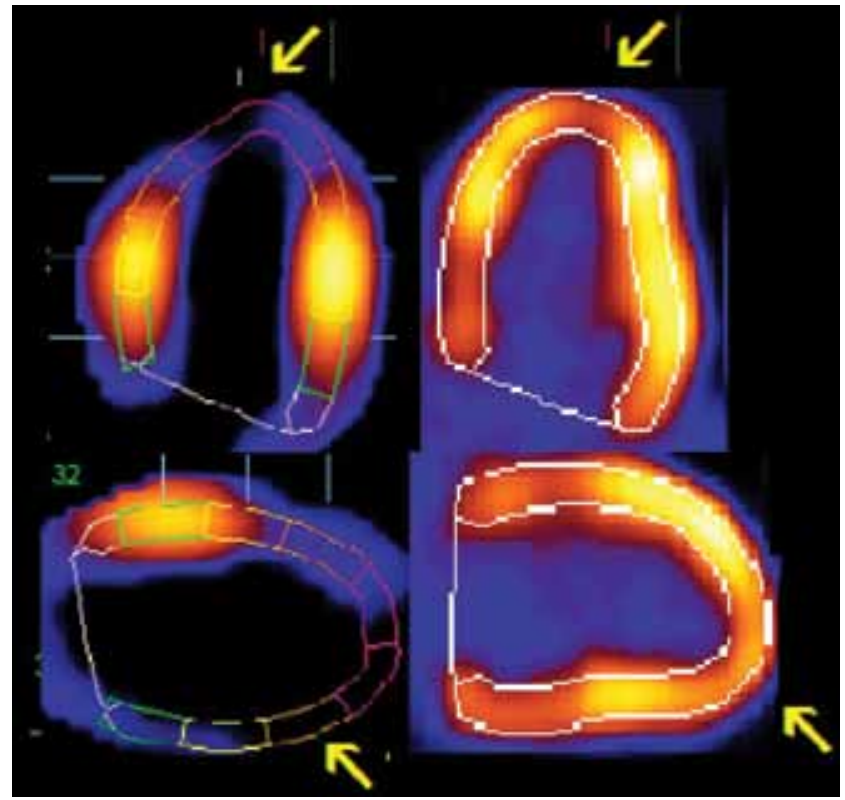

Figure 1. Viable myocardium uptake pattern. Comparison of 99mTc-MIBI perfusion (left column) and 18FDG uptake (right column) There is perfusion/metabolic mismatch, typical for myocardial hibernation. The extensive apical, periapical and posterior wall perfusion defect show high 18FDG uptake reflecting presence of glucose utilization and preserved myocardial metabolism

sion, while preserved glucose uptake- indicates the presence of severely impaired but viable myocardium (Figure 1). Myocardial segment which indicates normal or even decreased, but still present perfusion are naturally classified as viable (normal or hypoperfused myocardium). Those segments also maintain metabolism, but not necessarily show 18FDG uptake: the myocardial substrate demand is dependent on the metabolic and nutritional status (Figure 2A and B).

\section{Physiologic cardiac metabolism}

Normal cardiac muscle is able to produce energy from different substrates: free fatty acids (FFA), glucose and pyruvate. The proportion depends on the substrate delivery, hormone levels (mainly insulin) cardiac work load and perfusion. Different substrate utilization is observed in normal heart in fasting and postprandial stage. FFA are preferred substrate and account for up to $70 \%$ of cardiac energy metabolism - in fasting condition, in terms of normal oxygen supply (Figure 3B). The carbohydrate (glucose and pyruvate) metabolism reaches only $30 \%$ of cardiac muscle demand. The uptake of carbohydrates in fasting stage is generally low, but can be variable and heterogeneous (Figure 3A). On the contrary: in postprandial state glucose is a preferred energy source, while fatty acid oxidation is suppressed (Figure 4). Understanding those physiological changes in cardiac metabolism is crucial for proper metabolic 18F-FDG PET study interpretation. The lack of FDG uptake is suggesting not only the presence of non viable (scar tissue) but also utilization of substrates other than glucose. Therefore the perfusion and FDG metabolic study must be interpreted together.
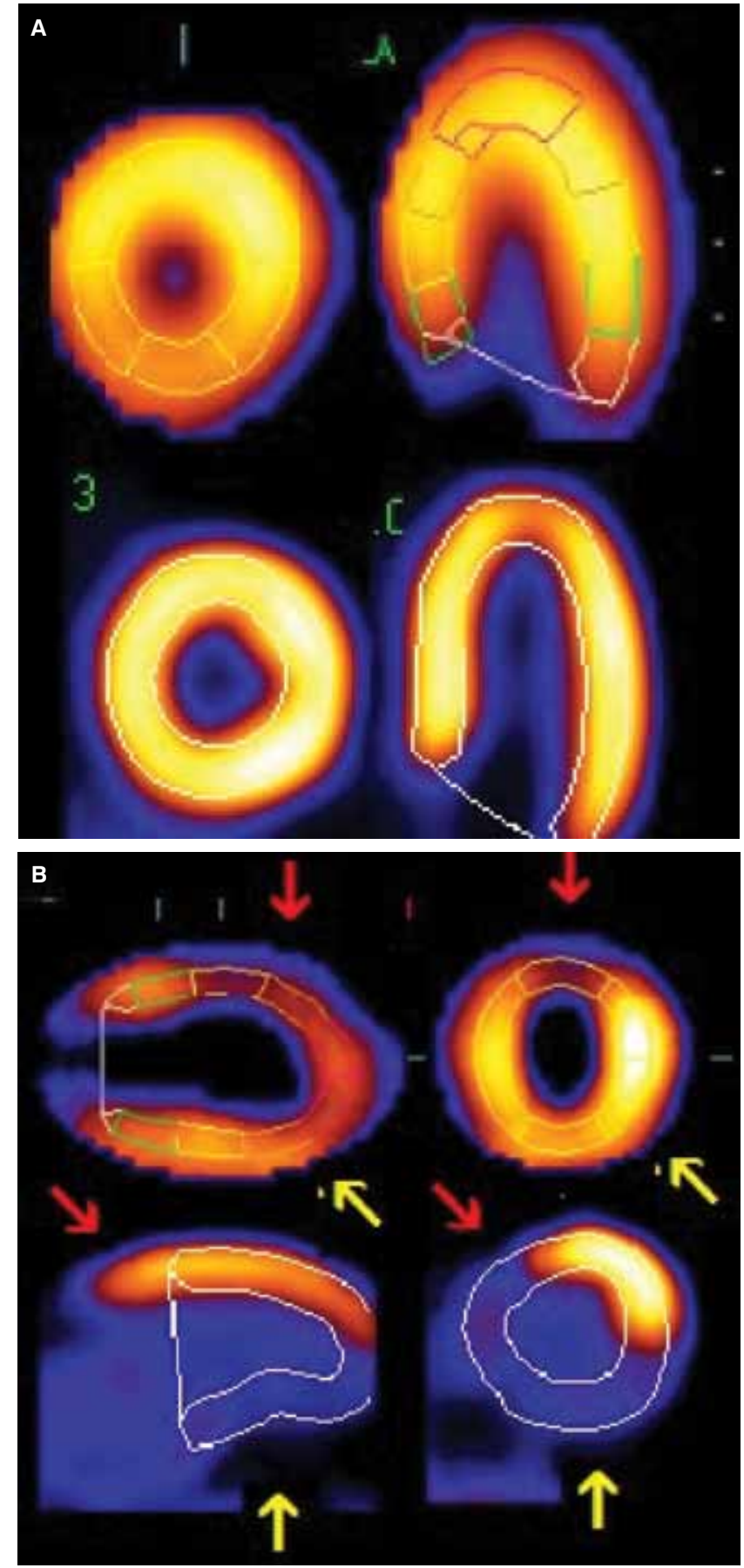

Figure 2. Viable myocardium uptake patterns: comparison of 99mTc-MIBI perfusion (upper rows) and 18FDG uptake (lower rows). A. There is a perfusion/metabolic match, typical for normal, viable myocardium. The picture show normal, homogenous perfusion and glucose metabolism. This perfusion/metabolic uptake pattern is typical for 18FDG glucose load protocol; B. This perfusion/metabolic mismatch - called "reversed mismatch" (posterior wall - yellow arrows) is consistent with presence of myocardial viability. The picture shows the presence of 99mTc-MIBI perfusion (upper row) with reduced 18FDG uptake (lower row). There is no $18 \mathrm{FDG}$ uptake in posterior wall, due to preferential FFA metabolism. Additionally there is also perfusion/metabolic mismatch in anterior wall, typical for myocardial hibernation (red arrows). This perfusion/metabolic uptake pattern is typical for 18FDG administration in fasting condition 


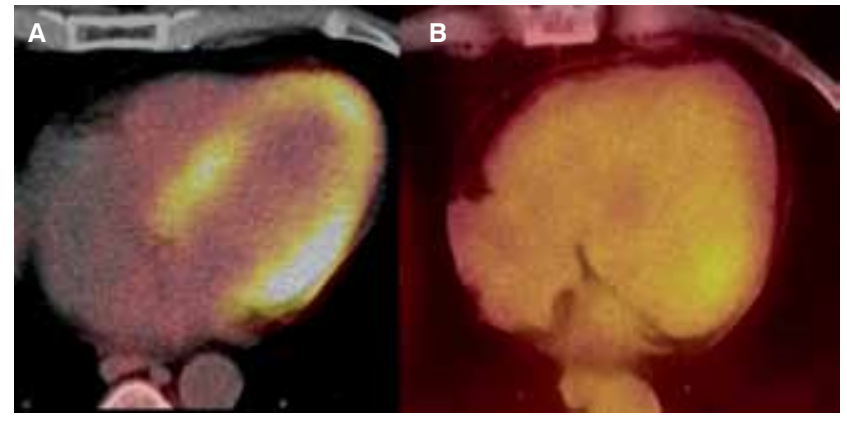

Figure 3. 18FDG PET/CT fusion images. Changes in FDG uptake in normal myocardium according to the nutritional status-fasting conditions. A. The heterogeneous non specific myocardial 18FDG uptake is not reflecting the myocardial pathologic condition. The interpretation is dependent on the myocardial perfusion; B. 18FDG uptake is low, due to relatively low glucose and insulin levels and high free fatty acids level

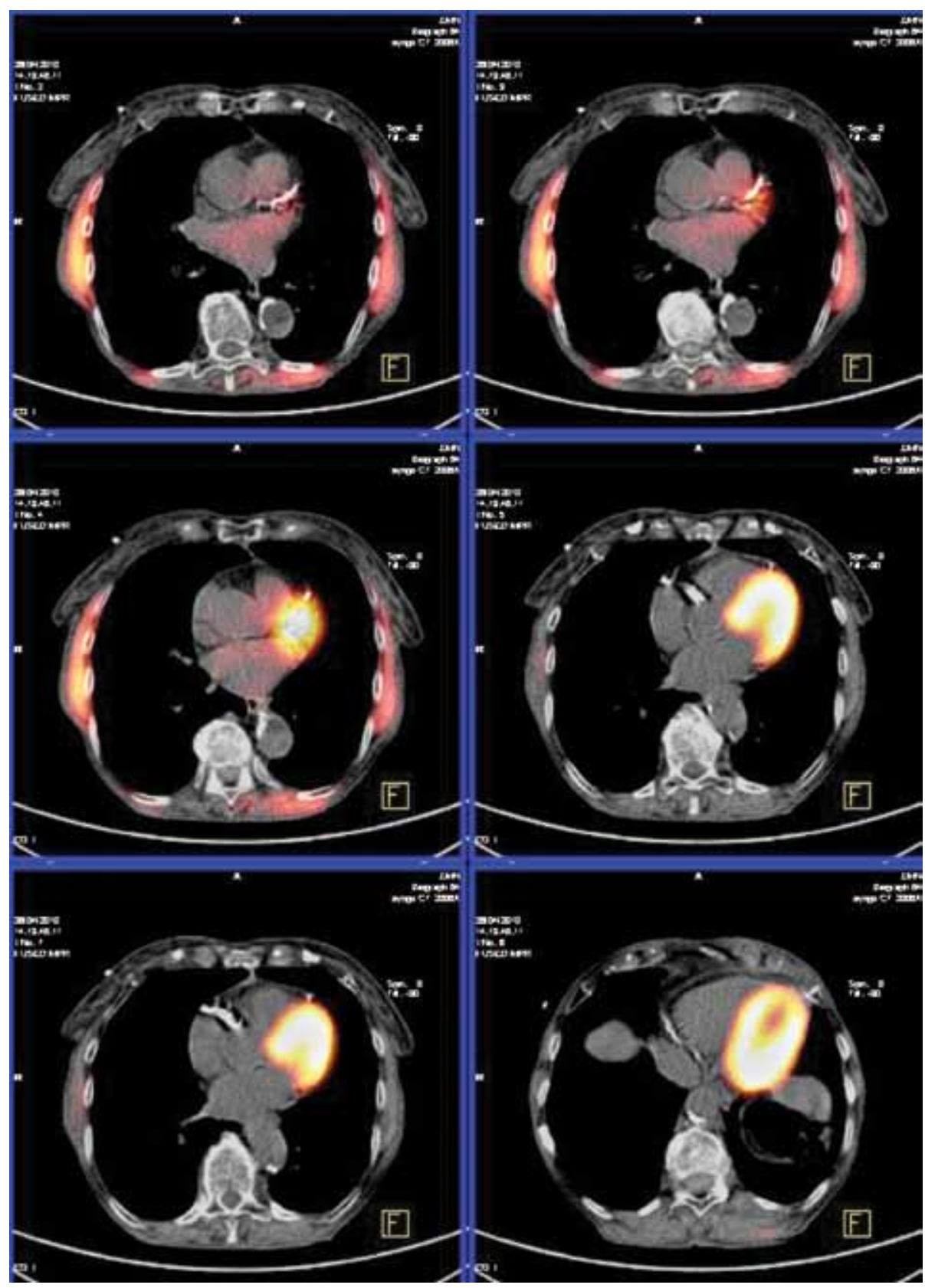

Figure 4. 18FDG PET/CT fusion images. Changes in FDG uptake in normal myocardium according to the nutritional status: postprandial conditions: $18 \mathrm{FDG}$ uptake is high due to facilitated glucose transport and utilization by normal myocytes, caused by release of endogenous insulin as an answer to increased plasma glucose 


\section{Cardiac metabolism in pathologic conditions}

There are several pathologic conditions altering the normal preferential FFA cardiac uptake normally observed in fasting condition. Ageing, cardiomyopathy (dilated and hypertrophic), ischemia, hibernation - are known reasons for preferential glucose utilization. On the contrary FFA are still preferentially used even in postprandial stage if patient is diabetic, insulin resistant or if high blood lipid levels are present.

\section{Ischemia, hibernation}

In the first line cardiac metabolic response to the acute disturbance such as ischemia is to switch myocardial substrate use from FFA to oxidation of glucose, lactate and glycogen, to maintain sufficient fuel supply during increased workload. Therefore on PET scan acquired in the fasting condition, glucose is preferentially accumulated in ischemic myocardium, and appears as a "hot spot" due to preferential FFA utilization by the rest of normal, non-ischemic myocardium (Figure 2B). Myocardial uptake of $18 \mathrm{~F}-\mathrm{FDG}$ is reflecting not only aerobic but also anaerobic glycolytic flux [6]. In normally functioning myocardium all available substrates for energy production are metabolized via oxygen dependent pathways. Only the hypoperfused myocardium is forced to produce energy via non oxidative metabolism. Therefore two cardiac FDG PET study protocols should be considered: Analysis of cardiac FDG uptake in fasting stage (fasting protocol) should be performed if acute ischemia is suspected. This protocol is preferred also for detection of metabolic changes due to prior ischemia in myocardium with apparent normal contractile function (metabolic memory phenomenon, after stress inducted ischemia). Assessment of cardiac viability while myocardial hibernation is suspected, demands to force the myocardium for primarily glucose utilization and registration of cardiac images in postprandial stage. Thus, to identify viable myocardium in mechanical dysfunctioning areas glucose loading protocols are preferred, because viable cells will accumulate preferentially 18F-FDG, whereas necrotic (scar) tissue will not show the deoxyglucose uptake (Figure 5).

The following example present typical for hibernation metabolic and perfusion uptake pattern - 99mTc-MIBI SPECT/ 18FDG PET mismatch.

\section{Case}

66-years-old male who has a history of coronary artery disease, status post myocardial infarction involving the inferior and infero-lateral wall (Figure 6).

\section{SPECT Findings}

The dipiridamole-stress 99mTc-MIBI examination revealed severe perfusion defect in apical, distal segments of anterior wall, in inferior, infero-lateral and infero-septal wall. The defects in apical and anterior wall region show partial reversibility. Some border reversibility at rest is seen around the fixed defect in inferior and infero-lateral wall. The extent of the stress defect is calculated as 58 percent of the left ventricle, the rest perfusion defect is covering $43 \%$ of the left ventricle.

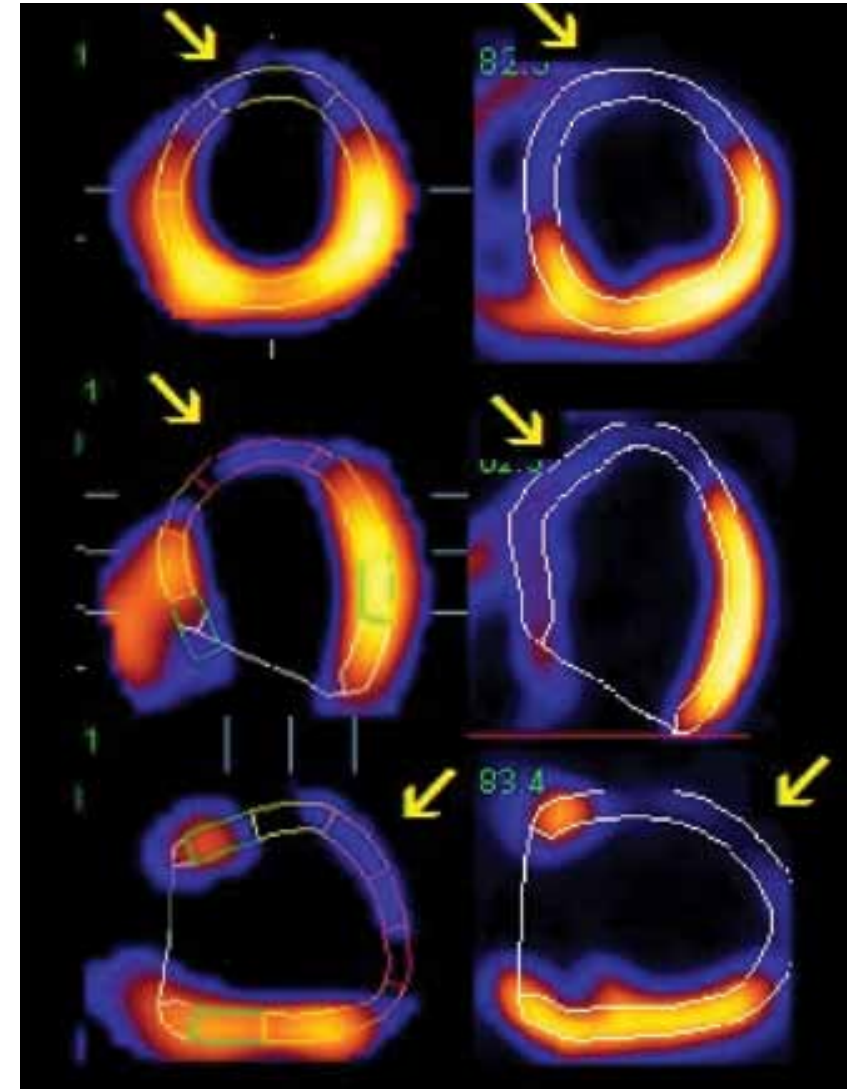

Figure 5. Comparison of 99mTc-MIBI perfusion (left column) and 18FDG uptake (right column) There is perfusion/metabolic match, typical for scar tissue (yellow arrows). The picture shows severe, extensive perfusion and glucose metabolism defect. This perfusion/metabolic uptake pattern is typical for 18FDG glucose load protocol, where the facilitated glucose transport causes the "glucose switch" and preferential glucose uptake by whole viable cardiac muscle

\section{SPECT Conclusions}

The rest and stress SPECT study revealed the transmural scar involving the apical, inferior, infero-lateral and infero-septal wall with borderline ischemia. Evaluation of myocardial viability is requested (Figure 7).

\section{FDG-PET findings}

There is slight inhomogenous, glucose metabolism seen in the whole left ventricular muscle.

\section{Impression}

There is viable, hibernated muscle present in the area matching the resting SPECT perfusion defect. The normal perfused myocardium shows glucose uptake similar to the hibernated area. The result of the PET/SPECT study reflex the muscle with preserved viability but compromised (Figure 8).

\section{Discussion}

Assessment of cardiac viability while myocardial hibernation is suspected, demands to force the myocardium for primarily glucose utilization and registration of cardiac images in postprandial stage. To identify viable myocardium in mechanical dysfunctioning areas glucose loading protocols are preferred, because viable 


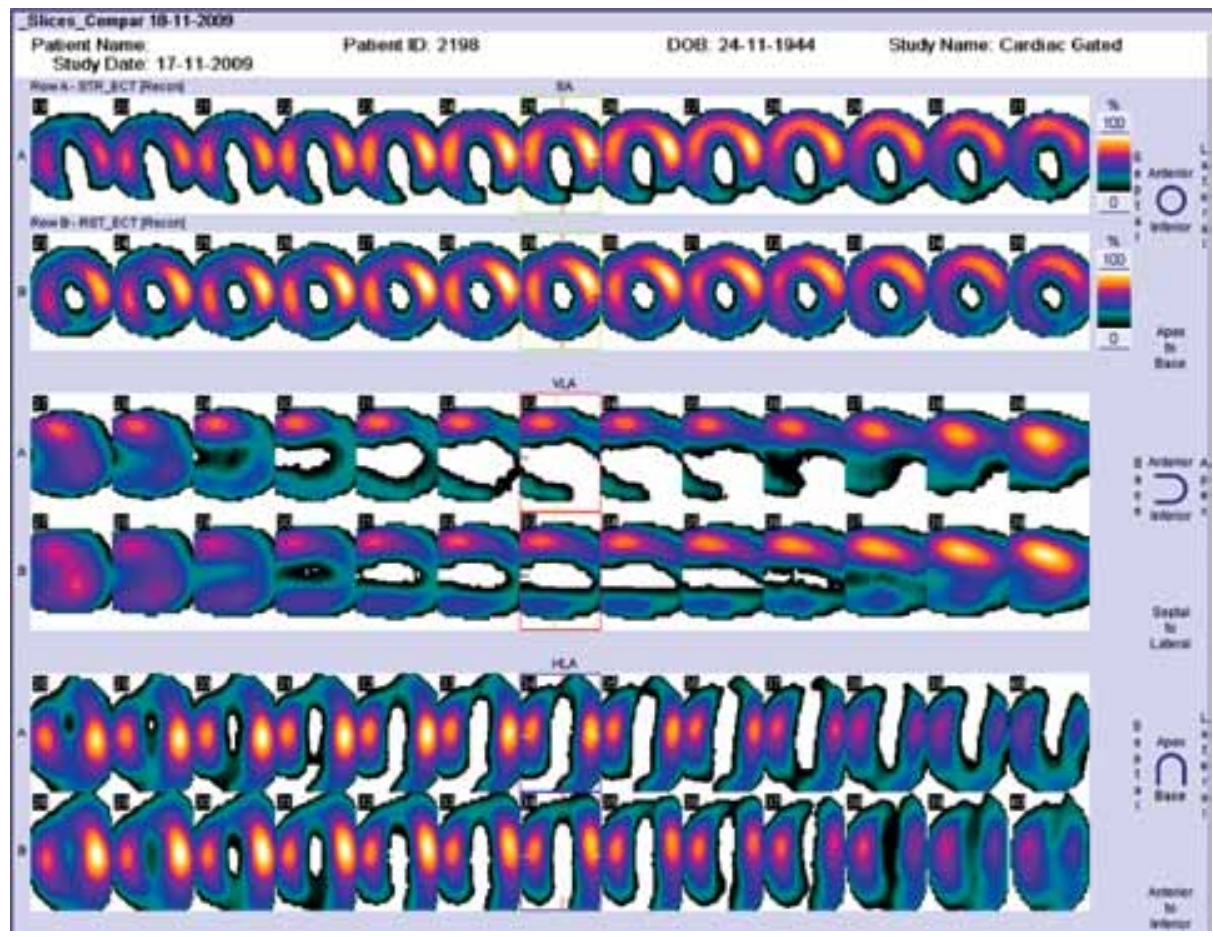

Figure 6. 99mTC-MIBI perfusion SPECT study. A. Upper rows: dipirydamole stress study; B. lower rows: rest study

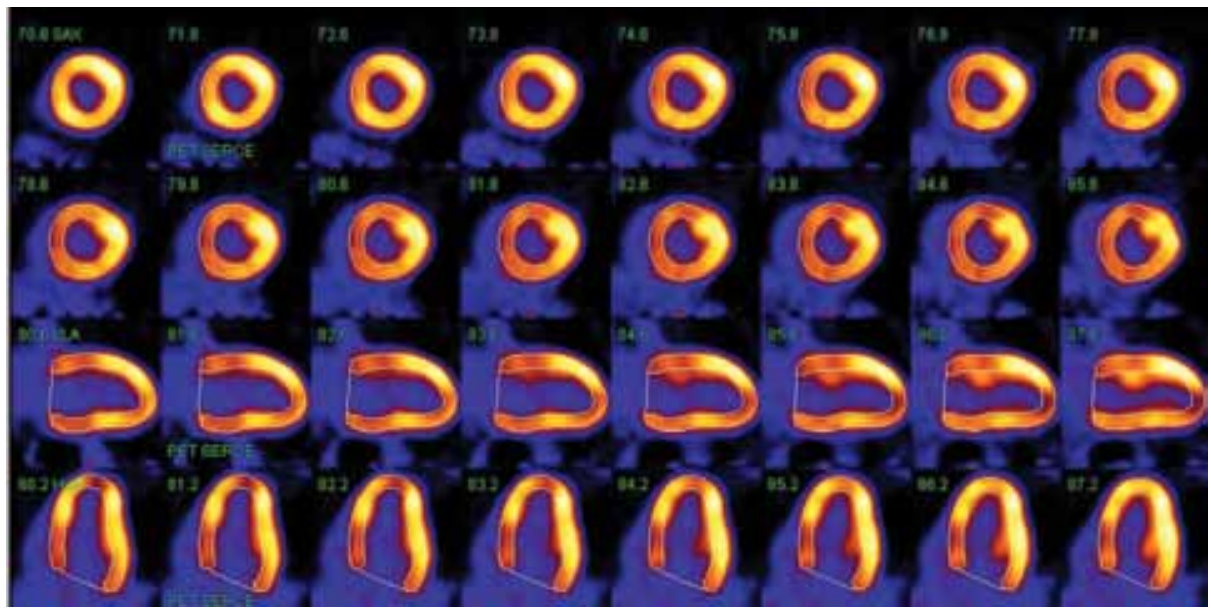

Figure 7. Glucose metabolism PET study with 18FDG

cells will accumulate preferentially 18F-FDG, whereas necrotic (scar) tissue will not show the deoxyglucose uptake. This case shows the FDG PET uptake pattern typical for adequate "glucose switch" after glucose load protocol. The study confirmed preserved viability in functional impaired myocardium.

\section{Dilated cardiomyopathy and left ventricular hypertrophy}

The cardiac hypertrophy or dilated cardiomyopathy are chronic diseases in which the cardiac metabolic changes occur at a transcriptional level. The genetic profile of the injured heart is switching for adaptation to a "fetal- like" profile that favors glu- cose over the FFA [7] (Figure 9). The current works are carried on the investigation of the connection between the cardiac hypertrophy and FFA oxidation impairment. It was confirmed that the activity of the process of myocardial FFA oxidation is an independent predictor of left ventricular mass in hypertrophy [8]. Not only the FDG-PET study, but also other radiopharmaceuticals allow for FFA oxidation measurements. The PET study with the palmitate - a long-chain fatty acid labeled with $11 \mathrm{C}$ is directly reflecting the activity of the FFA beta- oxidation. Studies on glucose and palmitate kinetics have shown that FFA uptake and metabolism is lower in cardiomyopatic patients than in controls [9]. The severity of this metabolic disturbance correlates good with the patient's clinical condition. 


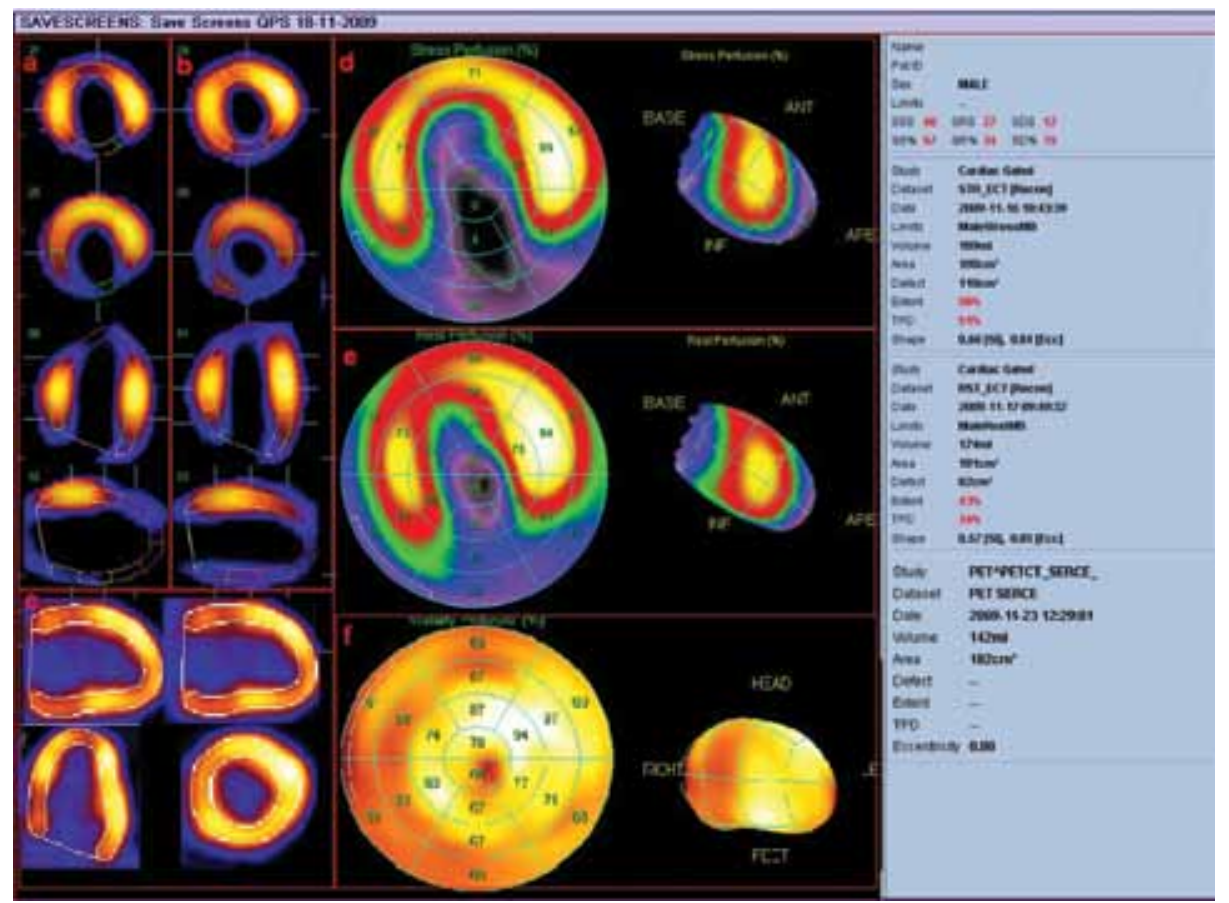

Figure 8. Evaluation of myocardial perfusion and metabolic activity: stress-rest single-photon emission computed tomographic (SPECT) myocardial perfusion imaging with 99mTc-MIBI and 18FDG PET metabolic imaging. The dipirydamole-stress 99mTc-MIBI perfusion study (A) shows the extensive, apical, antero-septal, distal anterior, inferior, infero-lateral, and infero-septal wall perfusion defect (58\% of the left ventricle). At rest (B), the perfusion defect shows partial reversibility, which reflect the transmural scar with the borderline significant stress inducted ischemia. A. $99 \mathrm{mTC}-\mathrm{MIBI}$ stress perfusion slices; B. 99mTc-MIBI rest perfusion slices; C. 18FDG-PET scan: cardiac glucose metabolism; D. Exercise 99mTc-MIBI perfusion polar map ("bulls eye" display); E. Rest 99mTc-MIBI perfusion polar map ("bulls eye" display); F. 18FDG polar map ("bulls eye" display)

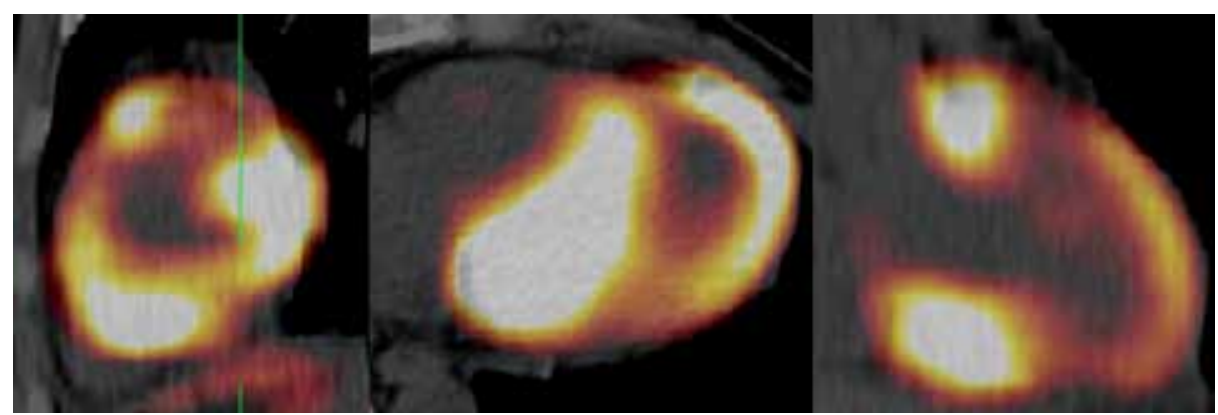

Figure 9. 18FDG PET/CT fusion images. Changes in FDG uptake in cardiomyopathy. The 18FDG uptake in hypertrophic myocardium is high, due to preferential uptake despite the fasting status and high FFA blood level

\section{Diabetes, obesity and insulin resistance}

In diabetic patients cardiac metabolism rely mainly on FFA as a substrate for energy production. Utilization of FFA meets 90$-99 \%$ of cardiac oxygen usage (MVO2). Normally in fasting stage and non-diabetic patients, FFA utilization is responsible for $70 \%$ MVO2, and glucose for the rest 30\% [10]. The normal cardiac ability to choose the substrates for energy production in cases of diabetes mellitus is impaired. In consequence, the diabetic heart in terms of increased myocardial work (e.g. stress) is unable increase glucose uptake and metabolism to maintain the adequate MVO2, this causes the higher sensitivity to ischemia (Figure 10, 11). Additionally the increased myocardial lipid accumulation may lead to cardiac cells injury - in consequence the apoptosis and electrical instability [11]. Increased body mass index (BMI) also influences the cardiac metabolism. It was shown, that FFA metabolism increases in obese patients. PET acetate and palmitate studies on young obese women confirmed the presence of cardiac metabolic shift toward FFA, which was positively correlated with worsening insulin resistance [12].

\section{Glucose load protocols}

There is no standard protocol for forced cardiac glucose uptake. The main goal is to shift cardiac metabolism to glucose utilization, by increasing the blood glucose level which is stimulating the endogenous insulin response and increase the intracellular glucose transport. The intravenous or oral glucose load may be used, the other possible choice is oral acipimox administration. The main goal of patient preparation, independent from the protocol 


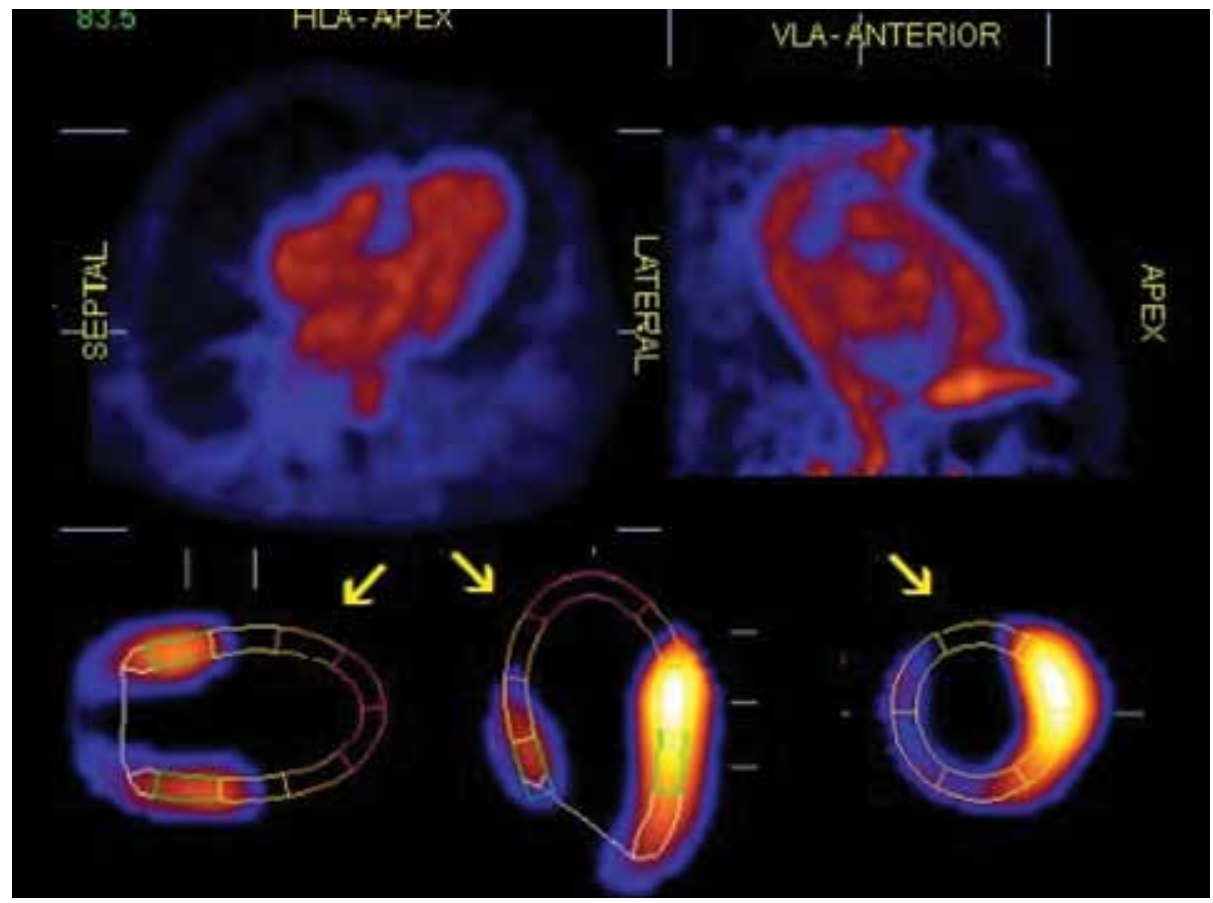

Figure 10. 18FDG PET and 99mTc-MIBI study: the 18FDG is present in the blood pool, there is no myocardial tracer uptake due to insulin resistance (upper row). The extensive perfusion defect shown on perfusion scan (lower row, yellow arrows) in this case can reflect both-scar tissue or hibernating myocardium. The result of the metabolic PET study make the perfusion study inconclusive

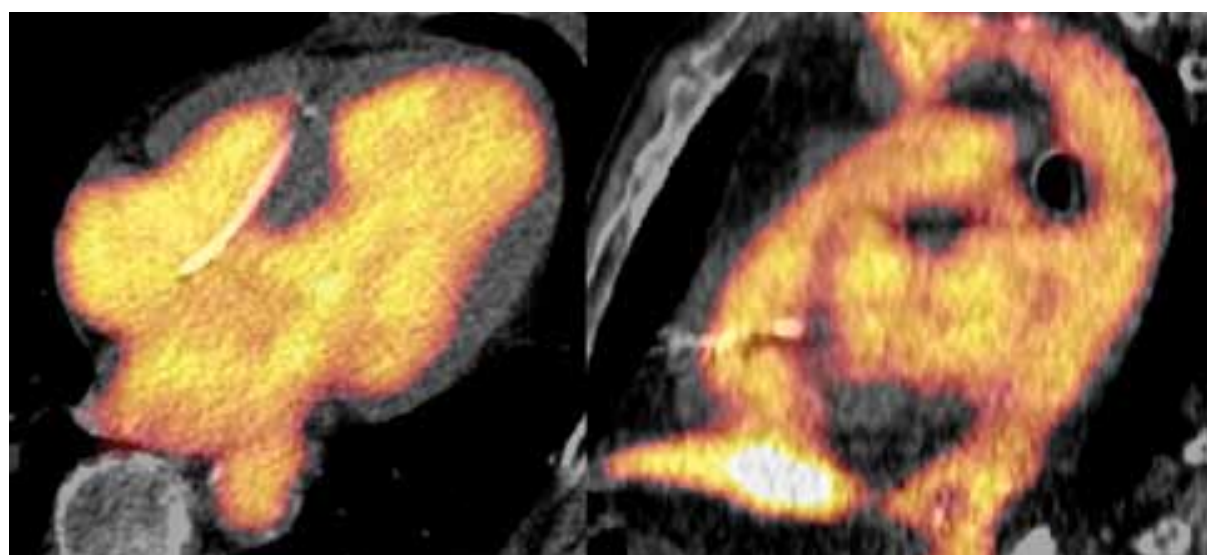

Figure 11. 18FDG PET/CT fusion images. No myocardial 18FDG uptake due to insulin resistance

- is to push the myocardium for preferential glucose utilization. All the protocols require keeping the patient fasting for at least $6 \mathrm{hr}$, This is necessary to induce proper endogenous insulin response. Shorter fasting time could depress this physiological process. A sample protocols are given below:

\section{Oral glucose}

This protocol is preferred in most nuclear medicine units because of its simplicity. The patient must be nondiabetic, with good blood glucose control, after at least 6 hours of fasting. For optimal FDG cardiac uptake the blood glucose has to be held at 100 to $140 \mathrm{mg} / \mathrm{ml}$ at FDG injection time. The protocol starts with oral glucose load of 25 to $100 \mathrm{~g}$. The blood glucose has to be measured periodically during the next $60 \mathrm{~min}$. The blood glucose level must fall sufficiently at $60 \mathrm{~min}$ after oral glucose administration. If the blood glucose ranges between
$100-140 \mathrm{mg} / \mathrm{dL}$, the FDG can be injected. The protocol is easy to implement and easy to perform in routine practice, but often needs some modification dependent on the blood glucose level. If the blood glucose rises over $140 \mathrm{mg} / \mathrm{dl}(140-160 \mathrm{mg} / \mathrm{dL})$ the $2 \mathrm{U}$ of regular insulin has to be additionally injected. The injected insulin amount should be further increased if the blood glucose reaches higher level. The amount of $1-2 \mathrm{U}$ insulin should be injected on every $10 \mathrm{mg} / \mathrm{dL}$ over the maximal allowed (140 mg/dL) blood glucose level [13].

\section{Intravenous glucose}

The intravenous glucose administration is more complicated but it avoids the problems with variable gastrointestinal glucose absorption time. This protocol can be used if the patient is non diabetic and his/her fasting blood glucose is less than $110 \mathrm{mg} / \mathrm{mL}$. The most convenient way is to use the hyperinsulinemic euglyce- 
mic clamp which is minimizing changes in the FFA, insulin and glucose levels, allowing for optimal myocardial 18F-FDG imaging. Still, despite the optimization, there is up to $10-15 \%$ of uninterpretable cases noted [6].

\section{Hyperinsulinemic-euglycemic clamp}

This protocol is effective both in diabetes and patients with normal glucose tolerance. It is used mostly in investigational studies, for exclusion of differences in glucose intestinal absorption. The protocol starts with the blood glucose level estimation, repetitive measurements should be carried on every 15 min until 18FDG injection. The insulin should be injected in declining infusion rates-starting with $0.16 \mathrm{U} / \mathrm{min} / \mathrm{kg}$ for 4 minutes, than $0.08 \mathrm{U} / \mathrm{min} / \mathrm{kg}$ for next 3 minutes and $0.04 \mathrm{U} / \mathrm{min} / \mathrm{kg}$ at 8 th minute. Co-infusion of $20 \%$ dextrose should be carried on in order to achieve the constant, euglycemic blood glucose concentration. The 18FDG is injected when blood glucose reach the range $80-110 \mathrm{mg} / \mathrm{dl}$ and remains constant for at least approximately $30 \mathrm{~min}$. For the best image quality, the insulin and dextrose infusion should be maintained for another $30 \mathrm{~min}$ [13].

\section{Glucose-insulin infusion}

The following protocol is often used both in diabetes and non-diabetes patients, it is less time consuming and less complicated than hyperinsulinemic euglycemic clamp. The protocol starts with the priming regular insulin injection - $6(10) \cup$, if the initial blood glucose level exceed 100 (140) mg/dl. The following co-infusions: insulin (100 U diluted in $500 \mathrm{ml} 0.9 \%$ saline solution) at a rate of $1,2 \mathrm{ml} / \mathrm{kg} / \mathrm{min}$ and $20 \%$ dextrose at a rate $1,8 \mathrm{ml} / \mathrm{kg} / \mathrm{h}$ should be carried on for next $60 \mathrm{~min}$. If the blood glucose level is maintained in the range of $80-140 \mathrm{mg} / \mathrm{dl}$, the $18 \mathrm{FDG}$ should be injected at $30 \mathrm{~min}[14]$.

\section{Acipimox oral administration}

Oral acipimox administration inhibits the peripherial lipolysis and thus decreases free fatty acids plasma level. The lack of this energetic substrate is causing the preferential glucose utilization by the myocardium (glucose switch). The usual protocol indicate the oral $250 \mathrm{mg}$ acipimox administration 90 to $60 \mathrm{~min}$ prior to 18F-FDG injection, after the overnight fasting [4]. Some protocols include additional carbohydrate-protein-rich meal after acipimox administration.

The more detailed description of the mentioned above protocols are described in guidelines published by the American Society of Nuclear Cardiology and the Society of Nuclear Medicine [15].

PET imaging has proved its accuracy and reproducibility for myocardial ischemia and viability assessment. However this unique in its ability for showing the particular substrate metabolism technique has unfortunately some disadvantages: PET imaging requires equipment and radiotracers synthesis onsite, some of them may not be widely available. The patient preparation is mandatory for optimization of diagnostic accuracy, the protocols are especially technically challenging in patients with metabolic disorders such as diabetes or insulin resistance. The technique has also comparatively lower spatial resolution in com- parison to the MRI study. Currently achieved PET resolution is 0.4 $\mathrm{cm}$, but the combined devices multislice computed tomography scanners with PET (PET/CT) are now sufficiently accessible in clinical practice. This combination allows for wider morphologic assessments: coronary calcium scoring and non-invasive coronary angiography may be added to myocardial perfusion/metabolic imaging if necessary.

\section{References}

1. Allman KC, Shaw LJ, Hachamovitch R, Udelson JE. Myocardial viability testing and impact of revascularization on prognosis in patients with coronary artery disease and left ventricular dysfunction: a meta-analysis. J Am Coll Cardiol 2002; 39: 1151-1158.

2. Pagley PR, Beller GA, Watson DD, Gimple LW, Ragosta M. Improved outcome after coronary bypass surgery in patients with ischemic cardiomyopathy and residual myocardial viability. Circulation 1997; 96: 793-800.

3. Di Carli MF. Myocardial viability Assessment with PET and PET/CT. In: Di Carli M.F, Lipton M.J. Cardiac PET and PET/CT imaging. Springer, 2007: 250-267.

4. Bax JJ, Veening MA, Visser FC et al. Optimal metabolic conditions during fluorine-18 fluorodeoxyglucose imaging: a comparative study using different protocols. Eur J Nucl Med 1997; 24: 35-41.

5. Udelson JE, Finley $\mathrm{J}$ et al. Comparison of imaging modalities in the assessment of myocardial Viability. In: Di Carli M.F, Lipton M.J. Cardiac PET and PET/CT imaging. Springer, 2007: 295-328.

6. Krivokapich J, Huang SC et al. Fluorodeoxyglucose rate constants, lumped constants and glucose metabolic rate in rabbit heart. Am J Physiol Heart Circ Physiol 1987; 252: H777-H787.

7. Gropler RJ. PET Measurement of Myocardial Metabolism. In: Di Carli MF, Lipton MJ. Cardiac PET and PET/CT imaging. Springer, 2007: 227-249.

8. de las Fuentas L, Herrero P, Peterson LR et al. Myocardial fatty acid metabolism: independent predictor of left ventricular mass in hypertensive heart disease. Hypertension 2003; 41: 83-87.

9. Davila-Roman VG, Vedala G, Herrero P et al. Altered Myocardial fatty acid and glucose metabolism in idiopathic dilated cardiomyopathy. J Am Coll Cardiol 2002; 40: 271-277.

10. Randle PJ, Priestman DA et al. Mechanisms modifying glucose oxidation in diabetes mellitus. Diabetologia 1994; 37: S155-S161.

11. Herrero P, Srinivasan M, Cassady DS et al. Impact of increased lipid delivery on myocardial fatty acid oxidation in type 1 diabetics. Circulation 2002; 106: II-478.

12. Delbeke $\mathrm{D}$, Lorentz $\mathrm{CH}$ et al. Estimation of left ventricular mass and infarct size from nitrogen-13-ammonia PET images based on pathological examination of explanted human hearts. J Nucl Med 1993; 34: 826-833.

13. Gerber BI, Ordoubadi FF et al. Positron emission Tomography using (18)FFluorodeoxyglucose and euglycaemic hyperinsulinaemic glucose clamp: optimal criteria for the prediction of recovery post ischaemic left ventricular dysfunction. Results from the europan community Concerted Action Multicenter study on use of (18)F-Fluorodeoxyglucose positon emission tomography for the detection of myocardial viability. Eur Heart J. 2001; 22: 1691-1701.

14. Martin WH, Jones R et al. A simplifid intravenous glucose lading protocol for fluorine 18fluorodeoxyglucose cardiac single-photon emission tomography. Eur J Nucl Med 1997; 24: 1291-1297.

15. Bacharach SL, Bax JJ, Case J et al. PET myocardial glucose metabolism and perfusion imaging with 18FDG, 13NH3 and 82Rb. Part 1. Guidelines for data acquisition and patient preparation. J Nucl Cardiol 2003; 10: 543-556. 